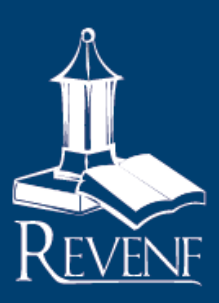

\title{
USO DE VENDAS ELÁSTICAS EN EL PRE Y TRANSOPERATORIO EN COLECISTECTOMIA PARA PREVENIR TROMBOSIS VENOSA PROFUNDA ${ }^{1}$
}

\author{
Carlos Mario Marín Rodríguez²
}

\author{
Institución:Universidad Latina de Costa Rica \\ Hospital de la Anexión, Guanacaste
}

\section{COMO CITAR}

Marín,CM. (2012).Uso de vendas elásticas en el pre y transoperatorio de una colecistectomía para prevenir la trombosis venosa profunda. [En línea].Rev. Enfermería Actual en Costa Rica, 22, 1-9 [citado (fecha)]. Disponible World Wide Web: 〈http://www.revenf.ucr.ac.cr/trombosis.pdf> ISSN 1409-4568

\section{RESUMEN}

Una de las intervenciones realizadas por el profesional de Enfermería para prevenir sucesos adversos en los pacientes sometidos a intervención quirúrgica, como es el caso de la colescistectomía, es la aplicación de vendas elásticas en los miembros inferiores en el pre y transoperatorio para prevenir la Trombosis Venosa Profunda (TVP). Sin embargo, esta práctica no ha sido fundamentada con la mejor evidencia disponible, por ello el objetivo de esta revisión es obtener un respaldo científico que avale esta práctica. Inicialmente, para recabar la mejor evidencia posible, se estableció una pregunta clínica en formato PICO; luego, se indagó en distintas bases de datos como PUBMED de las cual se obtuvieron 136 artículos siete de ellos de gran importancia clínica, ya que se halló, inclusive, una guía de práctica clínica. Luego se aplicó el análisis crítico mediante la plantilla CASPe y AGREE. Por último, a partir de los resultados encontrados se analizó la práctica que se desarrolla en el nosocomio con el fin de ser divulgados posteriorrmente y, así, mejorar la práctica clínica. Existe escasa o nula bibliografía específica que respalde el uso de vendas elásticas en el pre y transoperatorio de una colecistectomía, lo que puede deberse a que no hay evidencia suficiente respecto de sufrir trombosis venosa profunda por esta intervención. Por otro lado, cabe recordar que existen diferentes factores que afectan la colocación de las vendas elásticas, dígase la pericia del profesional que las aplica, su nivel profesional y la ausencia de métodos para medir la presión que se ejerce al colocar este tratamiento. Conclusión: no existe evidencia científica aparente que respalde el uso de vendas elásticas en los miembros inferiores aplicadas en el pre, trans y postoperatorio de una colecistectomía con la finalidad de prevenir la Trombosis Venosa Profunda, práctica que se lleva a cabo en el Hospital de La Anexión, Guanacaste.

Palabras clave: colecistectomía, práctica-enfermería, prevención, vendas-elásticas, trombosis-venosa-profunda

\footnotetext{
${ }^{1}$ Fecha de recepción: 22 de octubre del 2012

Fecha de aceptación: 22 de enero del 2012

${ }^{2}$ Estudiante de Enfermería de la Universidad Latina de Costa Rica. Hospital de la Anexión, Guanacaste.

Correo electrónico: carlosm258@ yahoo.com
} 


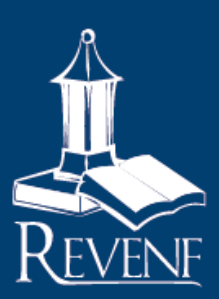

\title{
USING ELASTIC BANDS IN THE PRE AND TRANS-OPERATIVE CHOLECYSTECTOMY PREVENTION IN DEEP VEIN THROMBOSIS ${ }^{1}$
}

\author{
Institution: Universidad Latina de Costa Rica \\ Hospital Anexión, Guanacaste.
}

Carlos Mario Rodríguez Marín ${ }^{2}$

\section{CITED}

Marín,CM. (2012).Using elastic bands in the pre and trans-operative Cholecystectomy prevention in deep vein thrombosis. [En línea].Rev.

Enfermería Actual en Costa Rica, 22, 1-9 [cited (date)]. Disponible World Wide Web: <http://www.revenf.ucr.ac.cr/trombosis.pdf> ISSN 1409-4568

\begin{abstract}
One of the interventions by the nursing professional to prevent adverse events in patients undergoing surgery, such as the "cholecystectomy" is the application of elastic bandages on the legs in the pre and trans-operative to prevent Deep Vein Thrombosis (DVT). However, this practice has not been substantiated with the best available evidence. Therefore, the objective of this review is to obtain a scientific backing to support this practice. As part of the process of finding the best available evidence, established a clinical question in PICO format, subsequently developed a search strategy in different databases, resulting in PUBMED 136 articles, of which seven are clinical significance, including a clinical practice guideline. After critical analysis was applied using the template CASPe and AGREE. Finally,we analyzed the practice taking place in the hospital with the dissemination of the result in order to improvement of clinical practice. There is little or no specific literature supporting the use of elastic bandages in the pre and trans-operative Cholecystectomy for surgery, because there is no documented an increased risk of venous thrombosis due to this intervention. In addition, there are different factors affecting the placement of elasticbands and the professional expertise of the applications, their professional level and the absence of methods to measure the pressure exerted by placing this treatment. Conclusion: Thereis no apparent evidence to support the use of elastic bands in legs applied in Cholecystectomy surgery during the pre, trans and postoperative in order to prevent Deep Vein Trombosis, a practice that takes place in Anexión Hospital, Guanacaste.
\end{abstract}

Keywords: Bandages-elastic, Deep-veinthrombosis, Cholecystectomy, Practical-nursing, Prevention.

\footnotetext{
${ }^{1}$ Date of reception: October 22, 2012
}

Date of acceptance: January 22, 2012

${ }^{2}$ Student of Nursing in the University Latina of Costa Rica. Anexión Hospital, Guanacaste. Correo electrónico: carlosm258@yahoo.com 


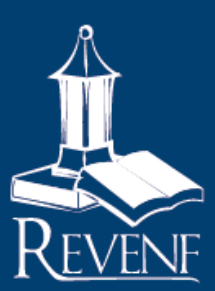

\section{INTRODUCCIÓN}

La colelitiasis o coledocolitiasis es descrita como una afección de las vías biliares que consiste en cálculos compuestos de colesterol y que se puede manifestar con las siguientes características:

1. Es más habitual en mujeres que en hombres.

2. su aparición tiende a observarse mayormente en personas de edades más avanzadas, esto se aplica en ambos sexos.

3. En mujeres la aparición es más precoz que en hombres.

4. La gran incidencia de litiasis en mujeres en edad fértil está relacionada con el número de embarazos. En nulíparas jóvenes, la enfermedad tiene una prevalencia semejante a la de los hombres de edad comparable. En cambio, la colelitiasis es mucho más frecuente en las multíparas. Este fenómeno depende del número de partos de la mujer más que de su edad. (Pontificia Universidad Católica de Chile, sf,sp)

Entre otros factores que pueden favorecer el desarrollo de cálculos biliares está el embarazo, debido a que el aumento de la motilidad vesicular, desde el primer trimestre, favorece la estasis de la bilis, la cual esta sobresaturada con colesterol, principalmente cuando se está en ayunas. Aunado a lo anterior, el sobrepeso, la obesidad y la descendencia indoamericana se consideran otro factor de riesgo en la aparición de cálculos. (Pontificia Universidad Católica de Chile, s.f.) Brunicardi (s.f.) menciona que algunos de los síntomas más comunes de esta enfermedad son: intolerancia a las comidas grasas, flatulencia, hinchazón abdominal, náuseas, vómitos

Respecto de los tratamientos de dicha patología, una de las formas de eliminarla es a través de una intervención quirúrgica, en este caso una colecistectomía, pero con ella pueden aparecer sucesos adversos, tales como complicaciones de las heridas, infección, cicatrices hipertróficas y queloides, eventración, complicaciones hemorrágicas, problemas anestésicos, complicaciones del tratamiento parenteral con líquidos, complicaciones respiratorias, complicaciones cardiovasculares, fístulas entero cutáneas, absceso residual intraabdominal( Aguila, s.f., s. p.)

Para prevenir estas complicaciones se han implementado una serie de prácticas que intentan disminuir el riesgo de aparición de dichos eventos. Algunas de estas estrategias conllevan el desarrollo de protocolos perioperatorios, cuya finalidad es disminuir los riesgos asociados a la cirugía, los cuales se pueden ver afectados por sesgos en su aplicación. No obstante, los avances científicos han demostrado la necesidad de modificar ciertas técnicas y prácticas en la atención.

Manterola (2002) comenta que la medicina basada en la evidencia tiene sus inicios en los años 30 con la propuesta de Louis y además menciona que desde los años 80 se ha manifestado propiamente el paradigma de la Medicina Basada en Evidencia, el cual ha sido responsable de crear una medicina más segura para los usuarios, basando el quehacer del profesional de la salud en una evidencia científica clara, oportuna y de buena calidad. 


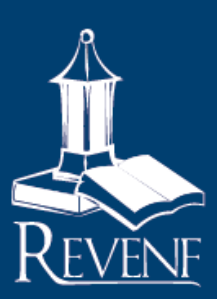

Tomando como base ese concepto nació una pregunta clínica con el propósito de buscar la mejor evidencia que garantice la seguridad del usuario (a) y además justifique el gasto económico que estas técnicas traen para lograr una atención con calidad y calidez.

Como mencionan Leteleir (2003) y Rojas ( 2011) la Medicina Basada en la Evidencia integra procesos a partir de la evidencia para así tomar la mejor decisión:

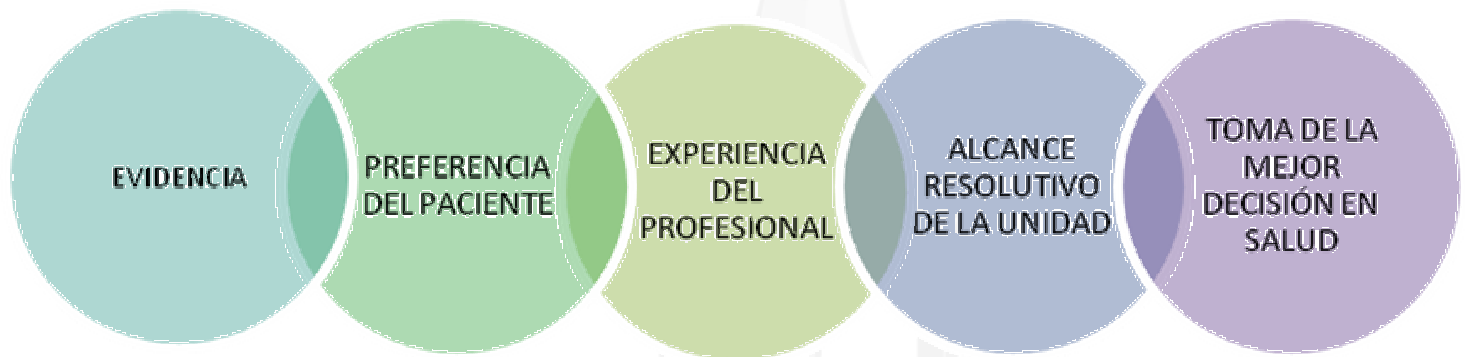

Este estudio tiene como meta fundamentar el uso de vendas elásticas en miembros inferiores en el pre, trans y postoperatorio como medida profiláctica para la aparición de Trombosis Venosa Profunda (TVP) en pacientes sometidos a Colecistectomia, ya que este es un protocolo que se utiliza en el Hospital de la Anexión, Guanacaste, Costa Rica. El objetivo mencionado se concretará mediante una revisión de los escritos científicos que hay sobre el tema en estudio.

La TVP es definida por la University of Maryland Medical Center (2011) como: "la formación de un coágulo sanguíneo en una vena que se encuentra en lo profundo de una parte del cuerpo, por lo general las piernas."(s. p.)

Para esta patología existen diferentes tratamientos desde el uso de vendas o medias hasta la utilización de heparinas de bajo peso molecular (HBPM). Martínez (2000) define las HBPM como un resultado de la heparina normal, pero con diferencias notables como el peso y algunas características propias.

Con el propósito de mejorar la atención a las personas sometidas a este tipo de intervenciones quirúrgicas, así como de proporcionarles seguridad y éxito tras la intervención, se revisa el material que existe sobre el tema, de manera que la atención esté basada en un fundamento científico y no empírico.

\section{MATERIALES Y MÉTODOS}

Para este estudio se aplicó la metodología para el desarrollo de la práctica clínica basada en la evidencia, la cual consta de las siguientes etapas:

1. Formulación de una pregunta de primera línea en el formato P.I.C.O.

2. Búsqueda de información científica disponible.

3. Análisis crítico de la información. 


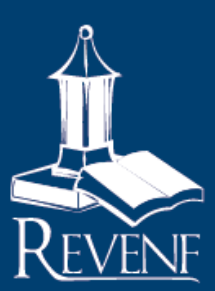

4. Implementación de los resultados encontrados en el quehacer diario del profesional.

5. Evaluación de la implementación.

La pregunta formulada se basa en el formato P.I.C.O. que se desglosa de la siguiente manera:

- Tipo de paciente o la patología de la que surge la pregunta $(P)$.

- La intervención que queremos analizar (I).

- La comparación con otra intervención (si procede) (C.)

- Los resultados clínicos esperados $(O)$.

Por cuanto la necesidad se plasma en la siguiente pregunta clínica:

En adultos conocidos sanos que se someten a una colecistectomía, ¿El uso de vendas elásticas en miembros inferiores en el pre, trans y postoperatorio inmediato disminuye el riesgo de sufrir de Trombosis Venosa Profunda?

En este estudio se realizó una búsqueda y revisión de artículos científicos, con el fin de identificar la mejor evidencia respecto del uso de vendas elásticas, de manera que se pueda contestar la pregunta clínica planteada.

Se utilizó el buscador GOOGLE para realizar la búsqueda y el administrador de artículos PUBMED. Se emplearon descriptores en inglés y español para la recuperación de resultados, los términos utilizados fueron ("Stockings, Compression"[Mesh] AND "Compression Bandages"[Mesh]) AND "Venous Thrombosis"[Mesh]) AND "Venous Thrombosis/prevention and control"[Mesh]

El resultado neto de este proceso fue una base de datos con 137 artículos, de los cuales en texto completo se lograron recuperar la mayoría. Sin embargo, del total de documentos recuperados únicamente 7 de ellos proporcionaron resultados que responden en forma parcial a la pregunta planteada acerca del uso de vendas elásticas en el pre, trans y posoperatorio de la colecistectomía.

De estos documentos, uno corresponde a una guía de práctica clínica, 3 son ensayos clínicos aleatorios y tres revisiones sistemáticas.

Los instrumentos aplicados para el análisis crítico de la información recuperada fueron el AGREE para Guías de Prácticas Clínicas y CASPe. En relación con el primero, su propósito es manifestado por Brouwer (2009) como la "evaluación de la calidad de las guías, proporcionar una estrategia metodológica para el desarrollo de las mismas y establecer qué información y cómo debe ser presentada en las guías” (p.7)

En el caso del segundo, como lo menciona Jiménez (2000):

Las preguntas incluidas en estos cuestionarios analizan en primer lugar la validez interna del estudio, en términos de adecuación y corrección metodológica [¿Son válidos los resultados del estudio]; en segundo lugar identifica cuáles son los resultados de la investigación [¿Cuáles son los resultados?] y en tercer lugar analiza si 


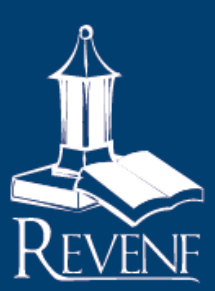

los resultados obtenidos en los estudios se pueden extrapolar a mi paciente [¿Me serán útiles los resultados para atender a mis pacientes?](s.p.)

\section{RESULTADOS}

Después de realizar la búsqueda y análisis de literatura que respondiera a la pregunta clínica que motivó este trabajo, no se encontró una evidencia concisa que demostrara la eficiencia de las vendas elásticas en el pre o transoperatorio. La evidencia encontrada se enfoca en investigaciones relacionadas con las medias de compresión y no con las vendas elásticas, pero se puede afirmar que son comparables, ya que su mecanismo de acción es el mismo al igual que la meta que persiguen.

Sí se encontró evidencia que respaldaba el uso de la vendas después de haber ocurrido una Trombosis Venosa Profunda, tal como lo menciona Snow et al (2007), quien afirma que después de ocurrido el evento en cuestión se iniciaría la terapia compresiva por un lapso mínimo de un año; por otra parte, el mismo autor menciona que los pacientes tienen una menor incidencia y severidad con solo la aplicación de las vendas en comparación con sus contrapartes que no se sometieron al tratamiento.

En la investigación de Aschwanden (2008) queda claro que la terapia compresiva después del evento reduce en un $40 \%$ las complicaciones, en otras palabras la evidencia presentada por Snow et al (2007) no difiere de esta.

Por otro lado, la evidencia presentada por Kahn et al (2007) menciona que las vendas reducen la hipertensión venosa, reflujo valvular, el edema, mejora la microcirculación y evita el daño de la epidermis. En ambos estudios se evidencia que el objetivo buscado fue disminuir el síndrome posttrombótico.

$\mathrm{Xu}$ (2010) explica que las vendas son una forma aceptable de disminuir el edema posterior a un evento trombótico -este estudio estaba dirigido a pacientes con Accidente Vascular Cerebral-; sin embargo, este autor recomienda no usarlas en personas que presentan este problema de salud por el alto riesgo de sufrir lesiones en la piel.

Por su parte, Kröger, K., Diehm, C., Moerchel, C. (2010), después de realizar una revisión sistemática de artículos, demostró que el vendaje elástico debe tenerse en cuenta con los pacientes de moderado a alto riesgo de sufrir una Trombosis Venosa Profunda (TVP), además enfatiza la necesidad de realizar un monitoreo de las piernas en busca de señales de daño de la piel causado por la presión del vendaje.

En el trabajo de Rocco (2006), queda evidenciado que el uso de vendas neumática es eficiente, además que su uso es muy parecido al de los fármacos; el autor explica que existe muy pocos efectos indeseados y secundarios al usarlas.

En el estudio de Dennis, M., Rudd A., Bowler, G. (2009) es muy claro que la mala aplicación de las técnicas podría producir la aparición de otros sucesos adversos, por ende, es necesario establecer un intervalo de presión, colocación y otros para disminuir el riesgo y aumentar los beneficios hacia el usuario. 


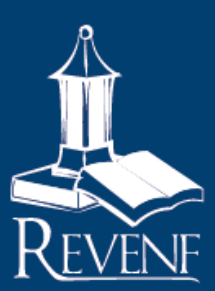

\section{DISCUSIÓN}

Después de desarrollar la presente revisión, el principal hallazgo fue concluir que no hay evidencias que asocien la trombosis venosa profunda y la cirugía de colecistectomía, lo cual implica la identificación de una práctica que se ha llevado a cabo durante muchos años en el nosocomio de la Anexión de Guanacaste.

Se destaca que dentro de la búsqueda realizada no se encuentra una asociación entre la trombosis venosa y la cirugía de colecistectomía, lo cual arroja luces sobre la necesidad de una investigación primaria que permita descartar esta práctica.

Por otro lado, se destacan las dificultades experimentadas durante el proceso de revisión y búsqueda de la literatura, dado que el acceso a buenas bases de datos es bastante costoso. Las bases de datos de acceso abierto no contienen suficiente evidencia que responda a la pregunta clínica planteada.

Es importante destacar que este proceso de investigación es una primera aproximación a encaminada a responder la pregunta planteada, por tanto, consistió en el primer ejercicio de práctica de enfermería basada en la evidencia.

Cabe recalcar que un gran número de estudios fueron elaborados con base en la aplicación de vendajes compresivos en el postoperatorio de las cirugías ortopédicas de cadera o rodilla y no en una colecistectomía, por ende, es importante realizar una investigación primaria con el fin de determinar el beneficio de la utilización de vendas elásticas en el pre, trans y postoperatorio de pacientes sometidos a colecistectomía y que no tienen antecedentes que aumenten el riesgo de presentar una Trombosis Venosa Profunda.

\section{CONCLUSIÓN}

No existe evidencia científica aparente que respalde el uso de vendas elásticas en los miembros inferiores aplicadas en el pre, trans y postoperatorio inmediato después de una colecistectomía con la finalidad de prevenir la Trombosis Venosa Profunda, práctica que se lleva a cabo en el Hospital de La Anexión, Guanacaste; tan solo hay evidencia del uso de vendas de compresión después de un evento trombótico. 


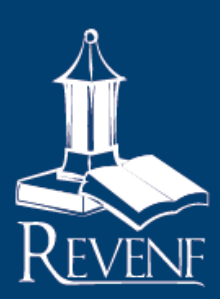

\section{REFERENCIAS}

Aguila, L.(s.f.) Complicaciones Posoperatoria. Recuperado

:http://sisbib.unmsm.edu.pe/bibvirtual/libros/medicina/cirugia/tomo i/Cap 26 Complicaciones\%20Postoperatorias.htm

Aschwanden, M. (2008). Effect of prolonged treatment with compression stockings to prevent post-thrombotic sequelae: A randomized controlled trial Journal of Vascular. Surgery. 2008 May; 47(5): 1015-21

University of Maryland Medical Center.(2011). Trombosis venosa profunda. Recuperado: http://www.umm.edu/esp ency/article/000156.htm

Brouwer, M. (2009). Instrumento para la evaluación de guías de práctica clínica Recuperado de: http://www.guiasalud.es/contenidos/documentos/Guias_Practica_Clinica/Spanish-AGREE-II.pdf

Brunicardi, F.(s.f.) Capítulo 31: Vesícula biliar y sistema biliar extrahepático.». Schwartz: Principios de cirugia ).México D.F, México : McGrawHill

Dennis, M., Rudd A., Bowler, G. (2009). Effectiveness of thigh-length graduated compression stockings to reduce the risk of deep vein thrombosis after stroke (CLOTS trial 1): a multicentre, randomised controlled trial. Lancet 2009 Jun 6373 : 1958-65. Recuperado: http://www.medscape.com/viewarticle/706670

Jimenez, J. (2000). Lectura crítica de la literatura científica (I): validez del estudio. Recuperado de http://www.elsevier.es/es/revistas/formacion-medica-continuada-atencion-primaria-45/lectura-critica-literaturacientifica-i-validez-estudio-9541-actualizaciones-2000

Jimenez, J. (2000), Lectura crítica de la literatura científica. II: Evaluación de los resultados. Recuperado de la página web:http://www.elsevier.es/es/revistas/formacion-medica-continuada-atencion-primaria-45/lectura-critica-literaturacientifica-ii-evaluacion-los-10860-actualizaciones-2000

Kahn, S. et al (2007). Effectiveness of compression stockings to prevent the post-thrombotic syndrome (The SOX Trial and Bio-SOX biomarker substudy): a randomized controlled trial. BMC Cardiovascular Disorders 2007, 7-21 DOI $10.1186 / 1471-2261-7-21$

Kröger, K., Diehm, C., Moerchel, C. (2010). Medizinische Thromboseprophylaxestrümpfe -Gibt es eine Evidenz? Dtsch Med Wochenschr 2011; 136: 276-279 DOI 10.1055/s-0031-1272524

Leteleir, L. (2003). Enfermería basada en evidencia. barreras y estrategias para su implementación. Recuperado:http://www.scielo.cl/scielo.php?pid=S0717-95532007000100003\&script=sci_arttext

Letelier, L. (2003) .La medicina basada en evidencia. Visión después de una década. Recuperado: :http://www.scielo.cl/scielo.php?pid=S0034-98872003000800016\&script=sci arttext\#af1 


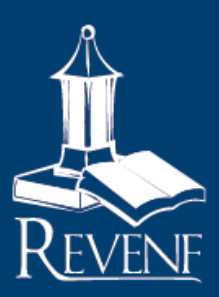

Revista Electrónica Enfermería Actual en costa Rica

www.revenf.ucr.ac.cr

Manterola, C.(2002), Medicina basada en la evidencia. Conceptos generales y razones para su aplicación en cirugía.Recuperado de la página wed:

http://www.cirujanosdechile.cl/Revista/PDF\%20Cirujanos\%202002 05/Rev.Cir.5.02.(21).pdf

Martínez, C.(2000). Relación riesgo-beneficio de las heparinas de bajo peso molecular en la profilaxis de la enfermedad tromboembólica venosa en cirugía general. Recuperado de página web:

http://www.aecirujanos.es/revisiones_cirugia/articulo_especial_septiembre2000.doc

Pontificia Universidad Católica De Chile (S.F.) Curso Integrado Medico Quirúrgico, Apuntes De Clases Del Capítulo De Gastroenterología. Recuperado:http://escuela.med.puc.cl/publ/temasgastro/Colelitiasis.html

Rocco, P.(2006). Foot pumps without graduated compression stockings for prevention of deep-vein thrombosis in total joint replacement: efficacy, safety and patient compliance. Int Orthop. 2008 June; 32(3): 331-336. DOI 10.1007/s00264-007$0326-9$

Rojas, L. (2011). Curso Bimodal Práctica Clínica de Enfermería Basada en la Evidencia: Alcances y Limitaciones. Rev. Enfermería Actual en Costa Rica [en línea].2011, No.20. Disponible World Wide Web: <http://www.revenf.ucr.ac.cr/ ebe.pdf> ISSN 1409-4568

Snow, V., et al (2007). Management of Venous Thromboembolism: A Clinical Practice Guideline from the American College of Physicians and the American Academy of Family Physicians.Ann Fam Med 2007;5:74-80. DOI 10.1370/afm.668

Xu, B. (2010). DVT in acute stroke The use of graduated compression stockings (Electronic version) Aust Fam Physician. 2010 Jun-Jul;39(6):485-7 